\title{
A Voxel-Based Diffusion Tensor Imaging Study of White Matter in Bipolar Disorder
}

\author{
Katie Mahon', Jinghui Wu', Anil K Malhotra',2,3, Katherine E Burdick ${ }^{1,2,3}$, Pamela DeRosse', \\ Babak A Ardekani ${ }^{4}$ and Philip R Szeszko*, ${ }^{*, 2,3}$ \\ 'Psychiatry Research, The Zucker Hillside Hospital, North Shore_-Long Island Jewish Health System, Glen Oaks, NY, USA; ${ }^{2}$ Feinstein Institute \\ for Medical Research, North Shore - Long Island Jewish Health System, Manhasset, NY, USA; ${ }^{3}$ Department of Psychiatry, Albert Einstein \\ College of Medicine, Bronx, NY, USA; ${ }^{4}$ Nathan S Kline Institute for Psychiatric Research, Center for Advanced Brain Imaging, Orangeburg, \\ NY, USA
}

There is evidence from post-mortem and magnetic resonance imaging studies that hyperintensities, oligodendroglial abnormalities, and gross white matter volumetric alterations are involved in the pathophysiology of bipolar disorder. There is also functional imaging evidence for a defect in frontal cortico-subcortical pathways in bipolar disorder, but the white matter comprising these pathways has not been well investigated. Few studies have investigated white matter integrity in patients with bipolar disorder compared to healthy volunteers and the majority of studies have used manual region-of-interest approaches. In this study, we compared fractional anisotropy (FA) values between 30 patients with bipolar disorder and 38 healthy volunteers in the brain white matter using a voxelwise analysis following intersubject registration to Talairach space. Compared to healthy volunteers, patients demonstrated significantly $(p<0.00$ I; cluster size $\geqslant 50$ ) higher FA within the right and left frontal white matter and lower FA within the left cerebellar white matter. Examination of individual eigenvalues indicated that group differences in both axial diffusivity and radial diffusivity contributed to abnormal FA within these regions. Tractography was performed in template space on averaged diffusion tensor imaging data from all individuals. Extraction of bundles passing through the clusters that differed significantly between groups suggested that white matter abnormalities along the pontine crossing tract, corticospinal/corticopontine tracts, and thalamic radiation fibers may be involved in the pathogenesis of bipolar disorder. Our findings are consistent with models of bipolar disorder that implicate dysregulation of cortico-subcortical and cerebellar regions in the disorder and may have relevance for phenomenology.

Neuropsychopharmacology (2009) 34, 1590-1600; doi: I 0.1038/npp.2008.2 I6; published online I4 January 2009

Keywords: bipolar disorder; white matter; diffusion tensor imaging; magnetic resonance imaging; tractography

\section{INTRODUCTION}

There is increasing evidence that white matter dysfunction may be an important consideration in the pathophysiology of bipolar disorder. Several studies reported white matter hyperintensities (WMH) in bipolar disorder (eg Aylward et al, 1994; Ahn et al, 2004), which appear to be one of the most robust and consistent neuroimaging findings in the literature (Norris et al, 1997; Stoll et al, 2000). More severe WMH have been linked to a greater number of hospitalizations and poorer treatment response (Moore et al, 2001). The presence of WMH may be indicative of an interruption in white matter fibers (eg Soares and Mann, 1997; Taylor

*Correspondence: Dr PR Szeszko, Psychiatry Research, The Zucker Hillside Hospital, North Shore — Long Island Jewish Health System, 7559 263rd Street, Glen Oaks, NY I 1004, USA, Tel: + I 718470 8489, Fax: + I 7|8343 1659, E-mail: szeszko@lij.edu

Received 20 March 2008; revised 29 October 2008; accepted 7 November 2008 et al, 2001), and/or an indication of early localized demyelination (Hajek et al, 2005).

Post-mortem and magnetic resonance imaging (MRI) studies have provided additional evidence for white matter abnormalities in the pathogenesis of bipolar disorder. In particular, altered expression of oligodendrocyte and myelin genes (Tkachev et al, 2003) and a lower density of oligodendroglial (Uranova et al, 2004) and glial (Rajkowska et al, 2001) cells have been reported in the prefrontal cortex of patients compared to healthy volunteers. MRI studies reported less left hemisphere (Kieseppa et al, 2003) and whole-brain (Strakowski et al, 1993; Davis et al, 2004) white matter volume in patients compared to healthy volunteers. Few studies have investigated regional differences in white matter using MRI, although Bruno et al (2004) identified lower white matter density in frontostriatal regions in bipolar patients compared to healthy volunteers using voxel-based morphometry.

A potentially more sensitive technique for the assessment of white matter integrity in bipolar disorder is the use of 
diffusion tensor imaging (DTI), which provides quantitative information regarding tissue water mobility. Fractional anisotropy (FA) is a measure of the coherence of white matter fibers derived from DTI, with higher FA potentially indicative of greater coherence. To date, few DTI studies have investigated the brain white matter in bipolar disorder. Furthermore, the majority of studies included small sample sizes and used a manual region-of-interest approach whose definitions may be potentially unreliable. In addition, findings have been inconsistent with reports of lower (Adler et al, 2004, 2006; Frazier et al, 2007; Bruno et al, 2008), higher (Yurgelun-Todd et al, 2007; Haznedar et al, 2005), or no differences (Beyer et al, 2005) in anisotropy between patients with bipolar disorder and healthy volunteers. A prior study that used tractography reported a significantly increased number of reconstructed fibers between the left subgenual cingulate and left amygdalohippocampal (AH) complex in patients with bipolar disorder compared to healthy volunteers without associated group differences in FA in the reconstructed fiber tract (Houenou et al, 2007).

Some studies suggest that it may be possible to examine the individual eigenvalues of the diffusion tensor to determine whether FA changes are associated with axial $\left(\lambda_{1}\right)$ or radial $\left(\lambda_{2}\right.$ and $\left.\lambda_{3}\right)$ white matter integrity (Song et al, $2002,2005)$. Specifically, axial diffusivity $\left(\lambda_{1}\right)$ is defined by how much diffusion occurs parallel to a given white matter tract whereas radial diffusivity $\left(\left(\lambda_{2}+\lambda_{3}\right) / 2\right)$ represents diffusion of water molecules perpendicular to the white matter tract. Previous work by Song et al (2002) demonstrated that white matter radial diffusivity was increased in shiverer mice with a dysmyelinated CNS, suggesting increased water mobility perpendicular to axons due to the lack of myelin sheath. In contrast, axial diffusivity was not affected in these animals suggesting intact axonal integrity. These findings were confirmed using electron microscopy. In addition, the extent of increased radial diffusivity reflected the severity of demyelination and following remyelination there was a concomitant decrease in radial diffusivity (Song et al, 2005). The investigation of axial and radial diffusivity could have implications for axonal and myelin-related pathologies, respectively and thus, may have relevance for better understanding FA alterations in the pathogenesis of bipolar disorder.

In this study we analyzed FA and axial/radial diffusivity data in a large cohort of patients with bipolar disorder and age- and sex-matched healthy volunteers using a voxelwise analysis. A potentially important advantage of a voxel-based approach is that abnormalities may be identified across the entire brain and with greater reliability compared to regionof-interest approaches. Some evidence suggests that white matter pathways connecting frontal cortical and subcortical structures, which are involved in mood regulation, may be disrupted in patients with bipolar disorder (Strakowski et al, 2005b). This disturbance may involve compromised bundle coherence in white matter connections subserving frontal areas, and/or a possible alteration in the myelination of these connective tracts. On the basis of this prior work, we hypothesized that FA would differ between groups in the frontal white matter and in the white matter surrounding subcortical areas. We further analyzed axial and radial diffusivity to ascertain whether FA differences might be associated with abnormalities in either axonal or myelinrelated integrity, respectively.

\section{MATERIALS AND METHODS}

\section{Subjects}

A total of 30 patients with bipolar disorder were recruited from The Zucker Hillside Hospital in Glen Oaks, NY. Diagnoses were based on clinical interview using the SCID for DSM-IV disorders (First et al, 1994) and supplemented by medical records and information provided by clinicians and family members, when available. All diagnoses were confirmed through a consensus conference involving psychologists and psychiatrists. The majority of patients had a diagnosis of Bipolar I Disorder $(n=25)$, whereas the remaining patients were diagnosed with either Bipolar II Disorder $(n=2)$, or Bipolar Disorder Not Otherwise Specified (NOS, $n=3$ ). All patients were being treated with antipsychotic medications and/or mood stabilizers. Eleven patients had a history of drug use (sedative abuse, $n=1$; sedative dependence, $n=2$; alcohol abuse, $n=5$; alcohol dependence, $n=3$; cannabis abuse, $n=3$; cannabis dependence, $n=6$; cocaine abuse, $n=3$; cocaine dependence, $n=2$; amphetamine abuse, $n=1$; amphetamine dependence, $n=2)$. A total of 38 healthy comparison subjects were recruited from the community through advertisements in local papers and word of mouth. Exclusion criteria for all study participants included any serious medical or neurological condition known to affect the brain. Exclusion criteria for healthy subjects also included any history of Axis I psychiatric illness as assessed by clinical interview (SCID-NP; First et al, 2001) or in first-degree relatives. This study was approved by the North Shore-Long Island Jewish Medical Center Institutional Review Board and written informed consent was obtained from all study participants.

\section{Handedness}

Handedness for all participants was assessed using a modified, 20-item version of the Edinburgh Inventory. A laterality quotient was computed for each participant according to the following formula: (Total $\mathrm{R}$-Total $\mathrm{L}$ )/ (Total $\mathrm{R}+$ Total L), where Total $\mathrm{R}$ and Total $\mathrm{L}$ refer to the total number of right- and left-hand items scored, respectively. Laterality quotients derived from this formula ranged from +1.00 (totally dextral) to -1.00 (totally nondextral). A laterality quotient of 0.70 or greater indicated a dextral classification for the subject, whereas subjects with a laterality quotient below 0.70 were classified as nondextral.

\section{Magnetic Resonance Imaging Procedures}

Magnetic resonance imaging examinations were conducted at the Long Island Jewish Medical Center on a GE $1.5 \mathrm{~T}$ system. A total of 26 DTI volumes were obtained from each subject that included 25 volumes with diffusion gradients applied along 25 nonparallel directions with $b=1000 \mathrm{~s} / \mathrm{mm}^{2}$ and $\mathrm{NEX}=2$, and one volume without diffusion weighting $(b=0 ; \mathrm{NEX}=2)$. Each volume consisted of 23 contiguous 
5-mm axial slices acquired parallel to the anterior-posterior (AC-PC) commissural line using a ramp sampled, spin-echo, single-shot echo-planar imaging (EPI) method (TR $=10 \mathrm{~s}$, $\mathrm{TE}=$ minimum $\mathrm{ms}, \mathrm{FOV}=22 \mathrm{~cm}$, matrix size $=128 \times 128)$.

To provide a high-resolution anatomical reference, 124 contiguous coronal images (slice thickness $=1.5 \mathrm{~mm}$ ) were acquired through the whole head using a three-dimensional Fast SPGR sequence with IR Prep $(\mathrm{TR}=10.1 \mathrm{~ms}, \mathrm{TE}=4.3 \mathrm{~ms}$, $\mathrm{TI}=600 \mathrm{~ms}, \mathrm{FOV}=22 \mathrm{~cm}$, matrix size $=256 \times 256)$ producing nominal in-plane resolution of $0.86 \times 0.86 \mathrm{~mm}^{2}$. In addition, an oblique axial fast spin-echo scan $(\mathrm{TR}=4 \mathrm{~s}$, $\mathrm{TE}=20 / 100 \mathrm{~ms}$, FOV $=22 \mathrm{~cm}$, matrix size $=256 \times 256)$ was acquired at the same slice positions as the diffusion tensor images and provided contiguous $5-\mathrm{mm}$ thick proton density (PD) and T2-weighted (T2) images. The fast spin-echo PD and T2 volumes were used for registration, distortion correction of diffusion images, and for image segmentation.

\section{Image Processing}

Image processing was conducted using previously published methods (Szeszko et al, 2005a, b, 2008). Briefly, nonbrain regions were removed from the SPGR images using the Brain Extraction Tool (Smith, 2002). Any nonbrain tissue that remained following the automatic brain extraction process was removed manually using MEDx (Sensor Systems Inc., MD, USA). The cropped SPGR images of all subjects were spatially normalized to the Montreal Neurologic Institute's 'Colin27' MRI volume (Holmes et al, 1998) using the nonlinear registration module of the Automatic Registration Toolbox (ART; Ardekani et al, 2005). For each subject, the SPGR volume was also registered to their fast spin-echo PD/T2 volumes using ART by a rigid-body six-parameter linear transformation (Ardekani et al, 1995). To correct for the spatial distortion in the DTI EPI images, the $b=0$ DTI volume was registered to the T2 volume using ART. Finally, we registered the FA, axial, and radial images to the stereotactic space of the 'Colin27' template by mathematically combining and applying the operations obtained from the three registration steps: (1) nonlinear within-subject mapping of the $b=0$ DTI volume to the T2 volume for distortion correction; (2) six-parameter linear within-subject mapping of the T2 to SPGR volume; and (3) nonlinear betweensubject mapping of the SPGR volume to the 'Colin27' reference volume. The accuracy of the registration algorithm used in this study has been demonstrated empirically to be superior to several others in reducing intersubject anatomical variability (Ardekani et al, 2005).

For each subject, we created a white matter mask in stereotactic space as follows. Using the six-parameter linear transformation obtained from the registration process, we resliced the cropped SPGR volume to the same orientation, voxel size, and matrix size as the PD/T2 volumes. First, the resliced cropped SPGR volumes were used as masks to delete the nonbrain regions from the PD and T2 volumes. Second, the resulting image sets (ie the cropped T2, PD, and resliced SPGR volumes) were used as three input channels to the FAST segmentation program (Zhang et al, 2001) to segment the brain into white matter, gray matter, and CSF. All individuals' white matter masks were then transformed to standard space using the above registration approach (minus the distortion correction step), averaged, and conservatively thresholded at $70 \%$ to obtain a white matter mask for the entire group. Voxelwise statistical analyses were confined to the voxels comprising this white matter mask.

For each subject an FA map was computed from the 26 DTI volumes following estimation of the diffusion tensor matrix and its eigenvalues for each voxel using methods described previously (Basser, 1995). The diffusion tensor and FA maps were computed in the original native coordinates of the acquired images before any registration operations were applied to the scalar values. The FA map of each subject was transformed into the standard 'Colin27' space as described above. There is evidence that the choice for a smoothing kernel size can influence study findings (Jones et al, 2005). It is difficult, however, to select unambiguously the size of the smoothing kernel in the absence of a priori information about the spatial dimensions of the effects of interest that may vary from one region to another. In the present study, we prospectively selected the smoothing kernel size to be approximately twice the spacing between voxels and for consistency with a prior study by our group (Szeszko et al, 2005a). Thus, both the registered FA and white matter images were smoothed with a threedimensional isotropic Gaussian kernel of FWHM $=3 \mathrm{~mm}$.

\section{Statistical Analyses}

Group differences in demographic variables were examined using independent groups $t$-tests or $\chi^{2}$-tests. FA values between groups were compared using voxelwise analysis of covariance with white-matter density (obtained from the segmentation process described above) and age as statistical covariates. FA values between patients and controls were considered significantly different at a given voxel if the null hypothesis in a two-tailed $t$-test was rejected at $p<0.001$ (Szeszko et al, 2005a, 2008). As a measure of false alarm rate, we estimated the false discovery rate (FDR) for the applied $p$-value threshold of 0.001 to be $q<0.015$ using the 'FDR' program of the FSL package with the conservative option, which estimates the FDR using the method of Benjamini and Hochberg (1995). As a further protection against multiple comparisons, significantly different FA values (corresponding to a $p$-value of 0.001 or less) were required to be part of a spatially contiguous cluster size of 50 voxels or greater. To confirm selected significant voxelwise findings (ie those that approximated the shape of a circle) circular regions of interest were placed on the raw FA maps in areas that differed significantly between groups and compared in SPSS using independent groups $t$-tests. To investigate the potential effects of substance use and the inclusion of Bipolar II and Bipolar NOS patients on the observed findings we performed several ancillary analyses in SPSS (version 11.5) after importing average FA values that differed significantly between groups from the voxelwise analysis. Specifically, patients without a substance use diagnosis $(N=19)$ and patients with a Bipolar I diagnosis $(N=25)$ were compared to all healthy volunteers, respectively. An additional analysis compared FA data between Caucasian patients $(N=18)$ and Caucasian healthy volunteers $(N=15)$; the only racial subgroup large enough for analysis. In these ancillary analyses FA was compared between groups using independent groups $t$-tests. 
Table I Sample Characteristics

\begin{tabular}{|c|c|c|c|c|c|}
\hline & Bipolar patients $(N=30)$ & Healthy volunteers $(N=38)$ & d.f. & Test statistics & $p$-value \\
\hline Age & $33.4(8.7)$ & $31.9(8.6)$ & 66 & $t=0.74$ & NS \\
\hline $\operatorname{Sex}(M / F)$ & 15/15 & $22 / 16$ & । & $\chi^{2}=0.42$ & NS \\
\hline Parental social class ${ }^{\mathrm{a}}$ & $2.8(1.1)$ & $2.6(0.86)$ & । & $t=0.88$ & NS \\
\hline Laterality quotient & $0.79(0.39)$ & $0.75(0.59)$ & 63 & $t=0.34$ & NS \\
\hline Race $^{\mathrm{b}}$ & $18,5,2,4,1$ & $15,14,2,6,1$ & 2 & $\chi^{2}=3.90$ & NS \\
\hline
\end{tabular}

Note. Data are presented as mean \pm SD in parentheses, unless otherwise indicated. There were data missing for the following variables: parental social class ( 1 patient), laterality quotient (2 patients, I healthy volunteer), and years of education (2 patients).

${ }^{a}$ Hollingshead Redlich Scale rated on a scale from I (highest) to 5 (lowest).

${ }^{b}$ Race coded as Caucasian, African American, Hispanic, Asian, and other. Because more than $20 \%$ of the categories for race had expected frequencies of less than 5 we combined the latter three groups (ie Hispanic, Asian, and other) into a single group for analysis.

In addition, radial, and axial diffusivity values for clusters that differed significantly between groups from the voxelwise analysis were imported into SPSS (version 11.5) for further analyses. Independent groups $t$-tests were used to compare patients and healthy volunteers in axial and radial diffusivity values for these clusters. Repeated-measures analysis of variance was used to investigate whether FA differences between groups could be attributed to specific abnormalities in axial or radial diffusivity. In these analyses, which were conducted separately for each FA cluster that differed significantly between groups, diffusivity (axial and radial) served as a within-subjects factor and group was the between-subjects factor. We also investigated whether FA along the length of extracted white matter tracts differed between groups using independent groups $t$-tests. In analyses investigating axial and radial diffusivity and FA along white matter tracts $\alpha$ was set to 0.01 (two tailed) to limit the possibility of a Type-I error.

\section{Tractography Procedures}

We used DTIStudio to perform tractography as described previously (Ashtari et al, 2007), which is based on the fiber assignment by continuous tracking method. A more detailed description regarding this program is provided elsewhere (Jiang et al, 2006). We used a threshold of 0.25 for FA values to perform tractography and discontinued tracking if the tract turning angle exceeded 0.70. We imported the clusters identified as significantly different between groups into the DTIStudio software program to extract and identify the white matter fibers that passed through them. Tractography was performed in template space on averaged DTI data from all individuals.

DTIStudio requires both a scalar field representing FA and a vector field representing the principal eigenvector of the diffusion tensor and thus, the average FA and principal eigenvector for all subjects were identified. Using the registration procedure described here and in our earlier work (Szeszko et al, 2005a,b, 2008) we obtained a deformation field for each subject that could be used for spatial normalization of the individual's DTI images to Talairach space. This deformation field is a vector-valued function and maps the coordinates of any given grid point in standardized space to its corresponding point in the individual's original DTI image space (Ardekani et al, 2005). To obtain the group-averaged FA image in Talairach space, we applied the deformation field of each subject to the FA image and computed the average of all spatially normalized FA images to yield the average FA in Talairach space for the group. The principal eigenvector field for the group was obtained by first computing the Jacobian matrix (Kaplan, 2003) of the deformation field at each grid point on the standardized space for all individuals. The eigenvector in each subject's original DTI image space that would be mapped to the particular grid point in the standardized space was then reoriented by premultiplying it with the computed Jacobian (Alexander et al, 2001). Reorientation of the principal eigenvector was completed for all individuals at all grid points in standardized space. Thus, at each grid point we obtained as many (reoriented) eigenvectors as there were individuals in the study. Lastly, for each grid point, a principal component analysis (Kent et al, 1980) was performed on the reoriented eigenvectors. The principal eigenvector of this analysis was then taken to represent the principal direction of water diffusion for the entire group of subjects at the grid point in question.

\section{RESULTS}

Patients did not differ significantly from healthy volunteers in distributions of age, sex, parental social class, handedness, race, or years of education (Table 1). An illustration of the intersubject registration of the FA maps for patients and healthy volunteers is provided in Figure 1 and the average SPGR images are provided in Figure 2. Voxelwise analysis of the white matter revealed three regions of higher FA in the right and left frontal white matter and one region of lower FA in the left cerebellum in bipolar patients compared to healthy volunteers (Table 2; Figure 3). Circular regions of interest placed on the raw FA maps in the left $(t=2.43$, d.f. $=66, p=0.02)$ and right $(t=1.94$, d.f. $=66, p=0.06)$ frontal white matter (corresponding approximately to clusters 2 and 3 , respectively) supported the voxelwise results. Independent group $t$-tests revealed significant group differences in radial diffusivity in clusters $1-3$ and axial diffusivity in clusters 1 and 4 (Tables 3 and 4). Effect sizes for group differences in axial and radial diffusivity 


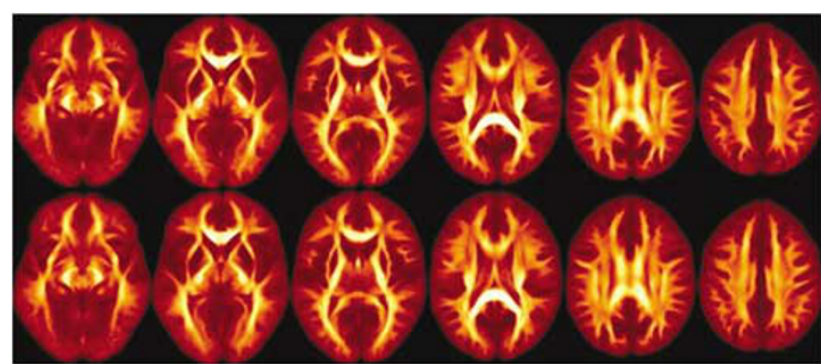

Figure I Average fractional anisotropy (FA) maps for patients (top row) and healthy controls (bottom row). Images from left to right correspond to $-5 \mathrm{~mm}$ below the anterior-posterior (AC-PC) plane and 5, 15, 25, 35, and $45 \mathrm{~mm}$ above the AC-PC plane.

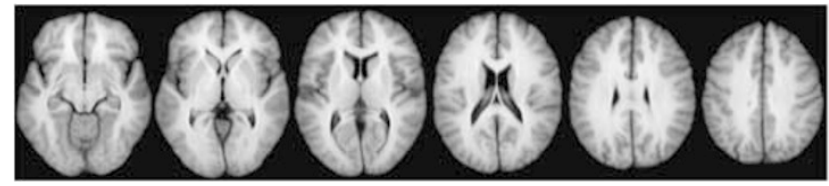

Figure 2 Average SPGR images corresponding to $-5 \mathrm{~mm}$ below the anterior-posterior (AC-PC) plane and 5, 15, 25, 35, and $45 \mathrm{~mm}$ above the AC-PC plane.

ranged from 3 to $18 \%$. Repeated-measures analysis of variance for axial and radial diffusivity measures revealed no significant group-by-region interaction for any of the four FA clusters that differed significantly between groups.

Two-dimensional images illustrating fibers passing through clusters 1-4 superimposed onto the color-coded average FA map are provided in Figure 4. Three-dimensional images illustrating fibers passing through these clusters are superimposed onto the average FA map in Figure 5. White matter passing through cluster 1 where FA was lower in patients compared to healthy volunteers appears to correspond to fibers of the pontine crossing tract. The white matter passing through clusters 2 and 3, where FA was higher in patients compared to healthy volunteers, appears to encompass fibers of the corticopontine tract (CPT), corticospinal tract (CST), and superior thalamic radiation fibers. Finally, white matter passing through cluster 4 appears to correspond to fibers of the superior longitudinal fasciculus (SLF). Visual inspection of fiber tracts indicates convergence of white matter from clusters 1-3 in the vicinity of the pontine crossing tract.

Average FA along the four sets of extracted white matter bundles was computed separately for patients and healthy volunteers. White matter FA along fibers of the pontine crossing tract (extracted from cluster 1) had significantly lower mean FA in patients compared to healthy volunteers along its length $(t=-3.32$, d.f. $=66, p=0.001)$. In addition, FA along fibers of the CST and CPT and thalamic radiation fibers extracted from cluster 2 was significantly higher in patients compared to healthy volunteers $(t=3.21$, d.f. $=66$, $p=0.002)$, but did not differ significantly between groups for fibers extracted from cluster $3(t=1.38$, d.f. $=66$, $p=0.172$ ). Lastly, white matter FA (extracted from cluster 4) along fibers of the SLF did not differ significantly between groups $(t=2.01$, d.f. $=66, p=0.048)$ following correction for multiple comparisons.
Ancillary analyses investigated the potential effects of substance use, having a diagnosis other than Bipolar I and race on the observed FA findings. Patients without a substance use diagnosis $(N=19)$ had significantly higher FA in the two right $(t=5.06$, d.f. $=55, p<0.001$ and $t=4.17$, d.f. $=55, p<0.001)$ and one left $(t=3.02$, d.f. $=55$, $p=0.004)$ frontal white matter regions as well as lower FA in the left cerebellum $(t=-3.30$, d.f. $=55, p=0.002)$ compared to healthy volunteers. Patients with a diagnosis of Bipolar I Disorder $(N=25)$ had significantly higher FA in the two right $(t=5.27$, d.f. $=61, p<0.001$ and $t=4.42$, d.f. $=61, p<0.001)$ and one left $(t=4.38$, d.f. $=61$, $p<0.001)$ frontal white matter regions as well as lower FA in the left cerebellum $(t=-4.04$, d.f. $=61, p<0.001)$ compared to healthy volunteers. In addition, restricting analyses to the subgroup of Caucasian subjects $(N=33)$ revealed significantly higher FA in the two right $(t=4.00$, d.f. $=31, p<0.001$ and $t=5.79$, d.f. $=31, p<0.001)$ and one left $(t=3.28$, d.f. $=31, p=0.003)$ frontal white matter regions as well as lower FA in the left cerebellum $(t=-2.51$, d.f. $=31, p=0.018)$ in patients compared to healthy volunteers.

\section{DISCUSSION}

These findings suggest that compared to healthy volunteers, adult patients with bipolar disorder have higher FA in the bilateral frontal white matter corresponding approximately to fibers of the CPT/CST and SLF as well as superior thalamic radiation fibers. In addition, FA was lower in the left cerebellar white matter, corresponding approximately to the pontine crossing tract, in patients compared to healthy volunteers. Although we found evidence for higher axial and lower radial diffusivity in regions of FA that differed significantly between groups, we did not find any evidence that group differences were associated with a specific deficit in either measure suggesting that both may contribute to white matter disturbances in bipolar disorder. The use of fiber tractography indicated that FA was significantly different in patients compared to healthy volunteers along the length of extracted white matter bundles comprising CPT and CST, thalamic radiation fibers as well as the pontine crossing tract. Moreover, convergence of fibers from the right and left frontal white matter along with those of the cerebellar region occurred in the pons, suggesting that an integrated network involving the cerebellum, striatum, and frontal cortex may be involved in the pathogenesis of bipolar disorder.

Few studies have assessed white matter integrity in bipolar disorder, especially using a voxelwise approach and thus, our findings are difficult to compare with prior work. Our findings converge with Haznedar et al (2005) who reported greater relative anisotropy in 40 patients with bipolar spectrum illnesses compared to $36 \mathrm{sex}$ - and age-matched healthy volunteers in the anterior frontal white matter using a region-of-interest approach. Yurgelun-Todd et al (2007) reported that 11 bipolar patients had significantly higher FA in the midline of the genu compared with 10 healthy controls. In contrast, Adler and colleagues reported lower FA in the superior frontal white matter using a region-of-interest approach in 11 medication-naive adolescent (Adler et al, 
Table 2 Mean Fractional Anisotropy Values for Patients and Healthy Volunteers

Talairach coordinates ${ }^{\mathrm{a}}$ (centroid)

\begin{tabular}{|c|c|c|c|c|c|c|}
\hline Brain region & $\begin{array}{c}\text { Bipolar patients } \\
(N=\mathbf{3 0})\end{array}$ & $\begin{array}{l}\text { Healthy volunteers } \\
\qquad(N=\mathbf{3 8})\end{array}$ & $\mathbf{x}$ & $\mathbf{Y}$ & $\mathbf{Z}$ & Cluster size $^{b}$ \\
\hline \multicolumn{7}{|l|}{ Left hemisphere } \\
\hline Cluster 2 & $338.36(39.48)$ & $300.90(32.87)$ & -24 & 3 & 24 & 83 \\
\hline Cluster 4 & $267.15(34.65)$ & $227.49(29.40)$ & 34 & -3 & 29 & 191 \\
\hline
\end{tabular}

Note. Data are presented as mean \pm SD in parentheses. Fractional anisotropy values are multiplied by a factor of 1000 .

${ }^{a}$ Talairach coordinates represent the centroid of the region.

${ }^{b}$ Cluster size is reported as number of voxels.

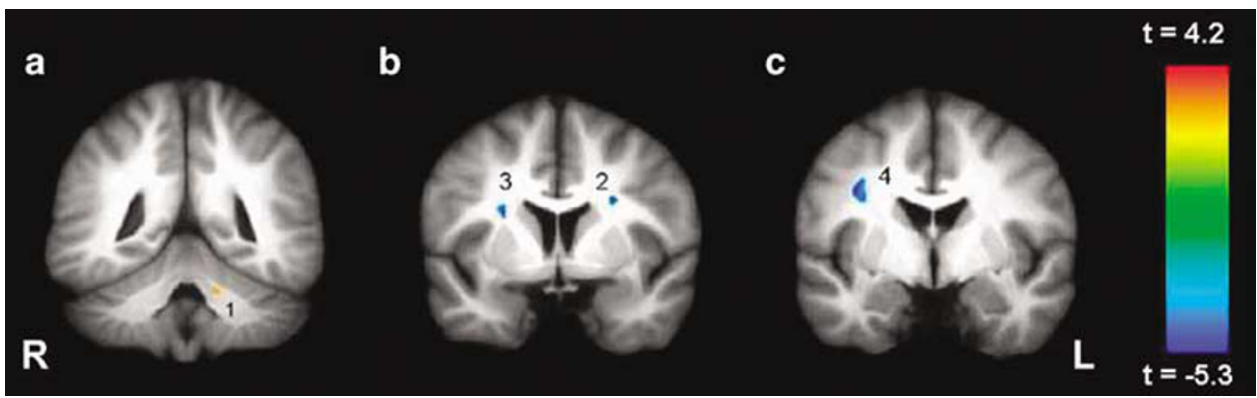

Figure 3 White matter regions where fractional anisotropy (FA) was significantly $(p<0.00$ I; 50 voxels) different in patients compared to healthy volunteers. A description of these regions can be found in the text and in Table 2.

Note: images are in radiologic convention.

Table 3 Mean Radial Diffusivity Values ${ }^{\mathrm{a}}$ for FA Clusters that Differed Between Patients and Healthy Volunteers

\begin{tabular}{|c|c|c|c|c|c|c|}
\hline Brain region & $\begin{array}{c}\text { Bipolar patients } \\
(N=\mathbf{3 0})\end{array}$ & $\begin{array}{l}\text { Healthy volunteers } \\
\qquad(N=38)\end{array}$ & $\begin{array}{c}\text { Test } \\
\text { statistics }\end{array}$ & d.f. & $p$-value & Effect size ${ }^{b}$ \\
\hline \multicolumn{7}{|l|}{ Left hemisphere } \\
\hline Cluster 2 & 2771.41 (129.54) & $2898.4 \mid(\mid 84.02)$ & $t=-3.20$ & 66 & 0.002 & 0.135 \\
\hline Cluster 4 & $2861.63(109.96)$ & $2938.80(135.12)$ & $t=-2.53$ & 66 & 0.014 & 0.089 \\
\hline
\end{tabular}

Note. Data are presented as mean \pm SD in parentheses.

Values are in units of $\mu \mathrm{m}^{2} / \mathrm{s}$.

${ }^{{ }^{b}}$ Effect size $=$ partial $\eta^{2}$.

2006) and 9 previously treated adult (Adler et al, 2004) patients with bipolar disorder compared to healthy volunteers. Beyer et al (2005) did not find any group differences in FA between 14 patients with bipolar disorder compared to 21 healthy individuals in the superior frontal, middle frontal, and inferior frontal white matter using a region-of-interest approach. In a voxelwise analysis of DTI data, Bruno et al
(2008) found a region of lower FA near the junction of the middle temporal and inferior gyri. Frazier et al (2007) reported that children with and at-risk for bipolar disorder have lower FA along the SLF compared to healthy controls, and that children with the disorder showed reduced FA in the cingulate-paracingulate white matter compared to both the atrisk and healthy control groups. Different findings among 
Table 4 Mean Axial Diffusivity Values ${ }^{a}$ for FA Clusters that Differed Between Patients and Healthy Volunteers

\begin{tabular}{|c|c|c|c|c|c|c|}
\hline Brain region & $\begin{array}{l}\text { Patients } \\
(N=30)\end{array}$ & $\begin{array}{l}\text { Healthy volunteers } \\
\qquad(N=\mathbf{3 8})\end{array}$ & Test statistics & d.f. & $p$-value & Effect size $^{b}$ \\
\hline \multicolumn{7}{|l|}{ Left hemisphere } \\
\hline Cluster 2 & $4746.60(230.53)$ & $4630.00(249.46)$ & $t=1.98$ & 66 & 0.052 & 0.056 \\
\hline Cluster 4 & $4229.08(149.54)$ & $4056.30(207.18)$ & $t=3.84$ & 66 & $<0.001$ & 0.183 \\
\hline
\end{tabular}

Note. Data are presented as mean \pm SD in parentheses.

${ }^{a}$ Values are in units of $\mu \mathrm{m}^{2} / \mathrm{s}$.

${ }^{\mathrm{b}}$ Effect size $=$ partial $\eta^{2}$
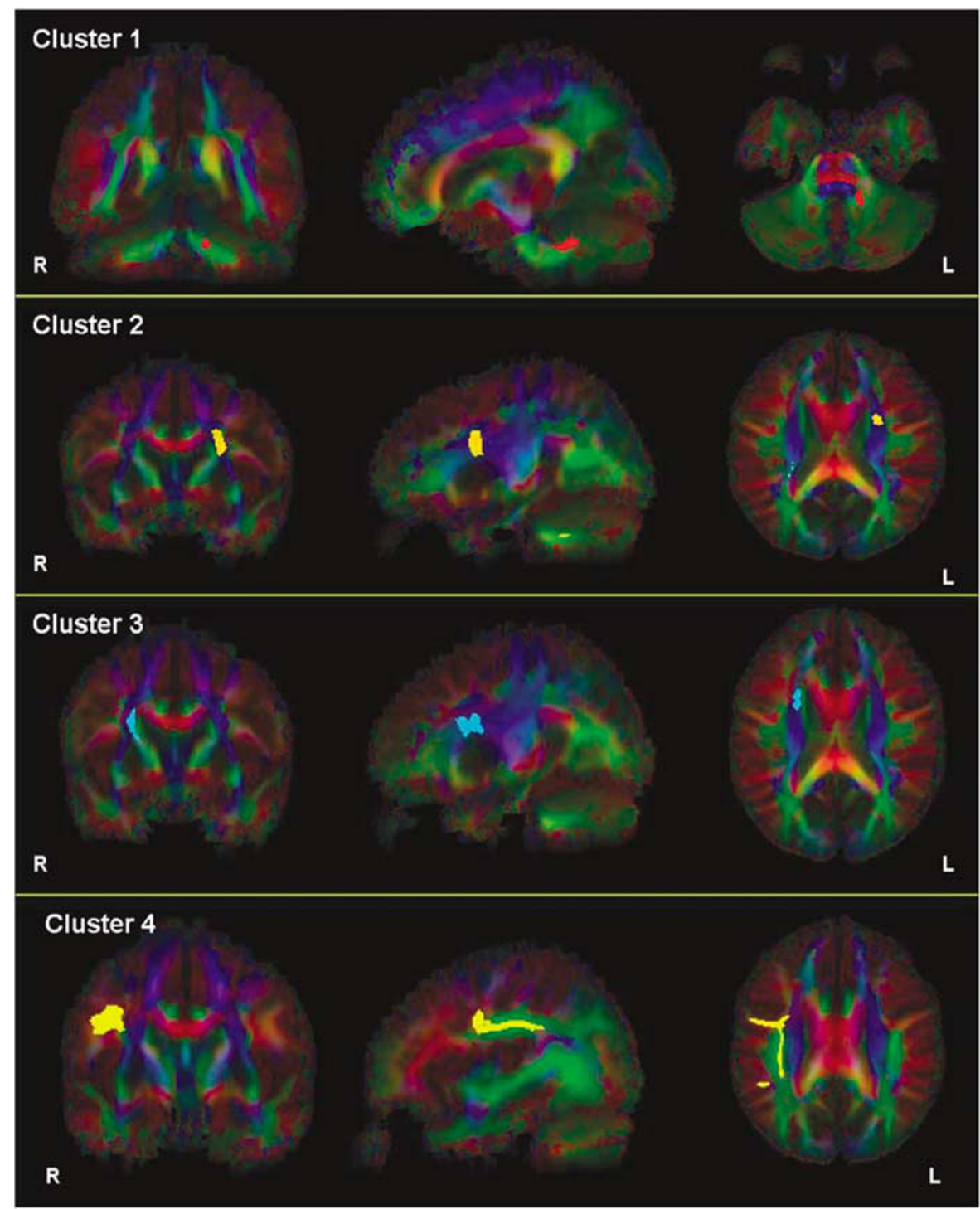

Figure 4 2D images illustrating fibers passing through clusters I-4, respectively, superimposed onto the color-coded average fractional anisotropy (FA) map of all subjects.

Note: images are in radiologic convention. Fibers passing through clusters I-4 are illustrated in red, orange, blue, and yellow, respectively. 


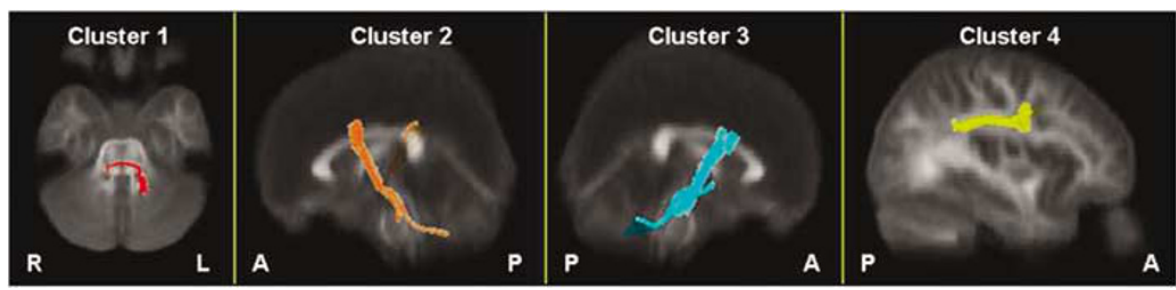

Figure $53 D$ images illustrating fibers passing through clusters $1-4$, respectively, superimposed onto the average fractional anisotropy (FA) map of all subjects.

Note: fibers passing through clusters I-4 are illustrated in red, orange, blue, and yellow, respectively.

studies may be related to sample size, differences in image acquisition and post-processing as well as the use of regionof-interest $v s$ voxel-based analysis approaches and diagnostic heterogeneity.

Although findings of higher FA in the frontal white matter among patients compared to healthy volunteers in our study most likely reflect an alteration in white matter connectivity, it is difficult to interpret the physiological significance of these findings, particularly in bipolar disorder because the pathophysiology is not well understood. One possible interpretation of the finding of higher FA in the frontal white matter is that it reflects greater coherence and/or directionality of white matter bundles in patients compared to healthy volunteers. In this regard we investigated whether group differences could be attributed to axonal or myelin-related properties of the white matter. Although significant group differences in axial and radial diffusivity were evident for some clusters of FA that differed between groups, we did not find support for preferential involvement of either diffusivity measure as a contributing factor to these white matter abnormalities. Inspection of the range of FA values observed in this study suggests that they may be in the vicinity of crossing fibers and thus, the finding of higher FA in patients could lead to the opposite interpretation, ie a loss of crossing fibers in patients. Similarly, Houenou et al (2007) reported a greater number of reconstructed fibers between the left $\mathrm{AH}$ and left subgenual cingulate in bipolar patients compared to healthy volunteers. As these authors point out, this may be related to an increase in strength and/or volume of the pathway between regions or, alternatively, a loss of orthogonal or crossing fibers in patients compared to healthy subjects, which may have permitted their fibertracking algorithm to work easier in patients to yield a concomitant greater number of fibers between regions of interest.

White matter bundles extracted from the clusters that differed significantly between groups corresponded approximately to the CPT, CST, pontine crossing tract and superior thalamic radiation fibers, and thus, are broadly supportive of models of altered cerebellar-striatal-prefontal connectivity in bipolar disorder (Green et al, 2007; Strakowski et al, 2005b). For example, mood dysregulation in bipolar disorder may result from deficits in frontal cortical modulation of cortical and subcortical structures involved in emotion generation (Green et al, 2007; Strakowski et al, 2005b). Recent evidence indicating that bipolar mania is characterized by reduced frontal inhibition of amygdala activity supports this hypothesis
(Foland et al, 2008). Other evidence from fMRI studies reported overactivation in frontosubcortical regions in patients with bipolar disorder (Blumberg et al, 2003; Chang et al, 2004; Strakowski et al, 2005a; Chen et al, 2006; Marchand et al, 2007; Wessa et al, 2007). Alterations in the white matter comprising cortical-subcortical pathways could thus conceivably be one mechanism of deficient modulation, although the relationship between FA and the BOLD response remains to be elucidated. Nevertheless, our data support the hypothesis for dysregulation in white matter comprising cortical and subcortical pathways, which may have relevance for the phenomenology of the disorder. For example, higher FA in the vicinity of the right SLF in patients with bipolar disorder may involve a large association pathway interconnecting right hemispheric brain regions (Makris et al, 2005) and the cognitive functions that they subserve, which may have relevance for the neuropsychology of bipolar disorder.

Higher FA in CST and CPT as well as superior thalamic radiation fibers in bipolar patients could have implications for serotonin synthesis, transport, and regulation throughout the brain (Alenina et al, 2006). Haddjeri et al (2000) describe a serotonergic feedback loop wherein stimulation of frontal cortical $5-\mathrm{HT}_{1 \mathrm{~A}}$ receptors inhibits the firing of serotonergic neurons in the pontine raphe nuclei. Abnormalities in the raphe nuclei have been documented in bipolar disorder (Baumann et al, 2002), as have abnormalities in the serotonin-transporter (5-HTT) system (eg Sun et al, 2001; Cannon et al, 2006) in both medicated and unmedicated patients. In addition to possible serotonergic implications, abnormalities along the fiber tracts extracted in our analysis may also have implications for abnormalities in noradrenergic transport. Some investigators reported abnormalities involving the locus coeruleus in bipolar patients (eg Baumann et al, 1999), a region of the pons that serves as a main source of noradrenergic transmission throughout the brain. Abnormalities in fiber pathways relevant to serotonergic and noradrenergic transmission, which may include the regions of higher FA observed in the present study, could have implications for the pathophysiology and pharmacotherapy of bipolar disorder.

The finding of abnormal FA in the left cerebellar white matter, which appears to be part of the pontine crossing tract, is consistent with the hypothesis that the cerebellum is important in psychiatric illness and mood regulation (Schmahmann, 2000; Strakowski et al, 2000; Konarski et al, 2005). In particular, a disruption in cerebellar and corticocerebellar connectivity has been proposed as a major neurobiological mechanism of emotional dysregulation 
(Schmahmann, 2000). Our findings converge with prior work implicating cerebellar abnormalities in first-episode bipolar patients compared to healthy volunteers (Adler et al, 2007). In addition, lower volume in the cerebellar vermis has been reported in bipolar patients who have undergone multiple affective episodes compared to firstepisode patients and healthy volunteers (Mills et al, 2005), suggesting that the cerebellum undergoes structural changes concomitant with illness progression. Consistent with this finding, Monkul et al (2008) reported that young male bipolar patients demonstrate a significant negative correlation between cerebellar vermal volume and number of previous affective episodes. Moorhead et al (2007) conducted a longitudinal tensor-based morphometry study and reported progressive cerebellar tissue loss over a 4 -year period in bipolar patients relative to controls. This tissue loss correlated inversely with the number of affective episodes that occurred during the 4-year study period. Interestingly, the region of significant loss in that study was a large cluster of gray and white matter in close proximity to the region of lower FA identified among patients in the present study.

There were several limitations to this study that should be acknowledged. The range of FA values reported here may suggest that clusters identified as abnormal may lie in regions of crossing fibers, and there is evidence that the single tensor model may not perform optimally in estimating $\mathrm{FA}$ in regions of fiber crossing and/or bending (Basser et al, 2000; Behrens et al, 2007; Kabasawa et al, 2008). In addition, it should be acknowledged that the anisotropic shape of the voxels and slice thickness may have biased FA and subsequent tractography analyses (eg Oouchi et al, 2007), especially for the cerebellar region. As with any voxelwise analysis there is the increased risk for a Type-I error, although we limited this possibility by restricting the analysis to the white matter and by using a conservative mask. Our patient sample was somewhat heterogeneous in terms of diagnosis, and included participants with a history of substance abuse, although ancillary analyses suggested neither of these was mediating factors. All patients were receiving psychotropic medications, however, and the potential effect of these medications on the brain white matter is largely unknown. In addition, we could not assess other possible factors that could influence white matter, including hydration and nutrition, and the potential clinical and cognitive correlates of these white matter abnormalities were largely unexplored. Although misregistration of images may be an issue with all VBM-style analyses, including the present study, we provided empirical data that our algorithm, in particular, minimizes this issue. Moreover, group effects were observed using manual region-of-interest placements, which were comparable to voxelwise analyses, suggesting that any misregistration of images did not appreciably affect study findings. It is also conceivable that the use of multiple NEX in our DTI sequence may have led to underestimation of motion artifacts (Smith et al, 2007) and the lack of cardiac gating may have led to additional artifacts (Skare and Andersson, 2001). Lastly, future studies could incorporate newly developed approaches to examining white matter tracts including the use of tract-based spatial statistics (Smith et al, 2006).
In sum, we report evidence for white matter abnormalities as assessed using DTI in a large cohort of patients with bipolar disorder compared to healthy volunteers. The use of tractography indicates that thalamic radiation fibers, and fibers of the corticospinal, corticopontine, and pontine crossing tracts may be involved in the pathogenesis of the disorder. Future studies could examine the relationship between white matter abnormalities in bipolar disorder and functional brain activity, as well as the cognitive and clinical correlates of these abnormalities.

\section{ACKNOWLEDGEMENTS}

This work was supported in part by grants from NARSAD, the Stanley Foundation and the NSLIJ Research Institute General Clinical Research Center (M01 RR018535).

\section{DISCLOSURE/CONFLICT OF INTEREST}

Ms Mahon, Ms Wu, Dr DeRosse, and Dr Burdick declare that except for income received from their primary employer no financial support or compensation has been received from any individual or corporate entity over the past 3 years for research or professional service and there are no personal financial holdings that could be perceived as constituting a potential conflict of interest. Dr Ardekani has received compensation from the North Shore-Long Island Jewish Health System, New York University School of Medicine, SUNY Albany, and SUNY Rockland. Dr Szeszko has received compensation from Boehringer Ingelheim. Dr Malhotra has received compensation from Bristol-Myers Squibb, Janssen Pharmaceutica, Roche Diagnostics, Vanda Pharmaceuticals, AstraZeneca, Eli Lilly, Pfizer, and PGX Health.

\section{REFERENCES}

Adler CA, Adams J, DelBello MP, Holland SK, Schmithorst V, Levine A et al (2006). Evidence of white matter pathology in bipolar disorder adolescents experiencing their first episode of mania: a diffusion tensor imaging study. Am J Psychiatry 163: 322-324.

Adler CM, DelBello MP, Jarvis K, Levine A, Adams J, Strakowski SM (2007). Voxel-based study of structural changes in first-episode patients with bipolar disorder. Biol Psychiatry 61: 776-781.

Adler CA, Holland SK, Schmithorst V, Wilke M, Weiss KL, Pan H et al (2004). Abnormal frontal white matter tracts in bipolar disorder: a diffusion tensor imaging study. Bipolar Disord 6: 197-203.

Ahn KH, Lyoo IK, Lee HK, Song IC, Oh JS, Hwang J et al (2004). White matter hyperintensities in subjects with bipolar disorder. Psychiatry Clin Neurosci 58: 516-521.

Alenina N, Bashammakh S, Bader M (2006). Specification and differentiation of serotonergic neurons. Stem Cell Rev 2: 5-10.

Alexander DC, Pierpaoli C, Basser PJ, Gee JC (2001). Spatial transformations of diffusion tensor magnetic resonance images. IEEE Trans Med Imaging 20: 1131-1139.

Ardekani BA, Braun M, Hutton BF, Kanno I, Iida H (1995). A fully automatic multimodality image registration algorithm. J Comput Assist Tomogr 19: 615-623. 
Ardekani BA, Guckemus S, Bachman A, Hoptman MJ, Wojtaszek M, Nierenberg J (2005). Quantitative comparison of algorithms for inter-subject registration of 3D volumetric brain MRI scans. J Neurosci Methods 142: 67-76.

Ashtari M, Cottone J, Ardekani BA, Cervellione K, Szeszko PR, $\mathrm{Wu} \mathrm{J}$ et al (2007). Disruption of white matter integrity in the inferior longitudinal fasciculus in adolescents with schizophrenia as revealed by fiber tractography. Arch Gen Psychiatry 64: $1270-1280$.

Aylward EH, Roberts-Twillie JV, Barta PE, Kumar AJ, Harris GJ, Geer $\mathrm{M}$ et al (1994). Basal ganglia volumes and white matter hyperintensities in patients with bipolar disorder. Am J Psychiatry 151: 687-693.

Basser PJ (1995). Inferring microstructural features and the physiological state of tissue from diffusion-weighted images. NMR Biomed 8: 333-344.

Basser PJ, Pajevic S, Pierpaoli C, Duda J, Aldroubi A (2000). In vivo fiber-tractography in human brain using diffusion tensor MRI (DT-MRI) data. Magn Reson Med 44: 625-632.

Baumann B, Bielau H, Krell D, Agelink MW, Diekmann S, Wurthmann C et al (2002). Circumscribed numerical deficit of dorsal raphe neurons in mood disorders. Psychol Med 32: 93-103.

Baumann B, Danos P, Krell D, Diekmann S, Wurthmann C, Bielau $\mathrm{H}$ et al (1999). Unipolar-bipolar dichotomy of mood disorders is supported by noradrenergic brainstem morphology. J Affective Disord 54: 217-224.

Behrens TE, Berg HJ, Jbabdi S, Rushworth MF, Woolrich MW (2007). Probabilistic diffusion tractography with multiple fiber orientations: what can we gain? Neuroimage 34: 144-155.

Benjamini Y, Hochberg Y (1995). Controlling the false discovery rate-a practical and powerful approach to multiple testing. $J$ R Stat Soc Ser B 57: 289-300.

Beyer JL, Taylor WD, MacFall JR, Kuchibhatla M, Payne ME, Provenzale JM et al (2005). Cortical white matter microstructural abnormalities in bipolar disorder. Neuropsychopharmacology 30: 2225-2229.

Blumberg HP, Martin A, Kaufman J, Leung H-C, Skudlarski P, Lacadie $\mathrm{C}$ et al 2003. Frontostriatal abnormalities in adolescents with bipolar disorder: preliminary observations from functional MRI. Am J Psychiatry 160: 1345-1347.

Bruno SD, Barker GJ, Cercignani M, Symms M, Ron MA (2004). A study of bipolar disorder using magnetization transfer imaging and voxel-based morphometry. Brain 127: 2433-2440.

Bruno SD, Cercignani M, Ron MA (2008). White matter abnormalities in bipolar disorder: a voxel-based diffusion tensor imaging study. Bipolar Disord 10: 460-468.

Cannon D, Ichise M, Fromm S, Nugent A, Rollis D, Gandhi S et al (2006). Serotonin transporter binding in bipolar disorder assessed using [11C]DASB and positron emission tomography. Biol Psychiatry 60: 207-217.

Chang K, Adleman NE, Dienes K, Simeonova DI, Menon V, Reiss A (2004). Anomalous prefrontal-subcortical activation in familial pediatric bipolar disorder: a functional magnetic resonance imaging investigation. Arch Gen Psychiatry 61: 781-792.

Chen C-H, Lennox B, Jacob R, Calder A, Lupson V, BisbrownChippendale $\mathrm{R}$ et al (2006). Explicit and implicit facial affect recognition in manic and depressed states of bipolar disorder: a functional magnetic resonance imaging study. Biol Psychiatry 59: 31-39.

Davis KA, Kwon A, Cardenas VA, Deicken RF (2004). Decreased cortical gray and cerebral white matter in male patients with familial bipolar I disorder. J Affect Disorders 82: 475-485.

First MB, Spitzer RL, Gibbon M, Williams JBW (1994). Structured Clinical Interview for DSM IV TR Axis I Disorders - Patient Edition (SCID-I/P). Biometrics Research Department, New York State Psychiatric Institute: New York.
First MB, Spitzer RL, Gibbon M, Williams JBW (2001). Structured Clinical Interview for DSM IV TR Axis I Disorders-Non-patient Edition (SCID-I/NP). Biometrics Research Department, New York State Psychiatric Institute: New York.

Foland LC, Altshuler LL, Bookheimer SY, Eisenberger N, Townsend J, Thompson PM (2008). Evidence for deficient modulation of amygdala response by prefrontal cortex in bipolar mania. Psychiatr Res 162: 27-37.

Frazier JA, Breeze JL, Papadimitriou G, Kennedy DN, Hodge SM, Moore CM et al (2007). White matter abnormalities in children with and at risk for bipolar disorder. Bipolar Disord 9: 799-809.

Green MJ, Cahill CM, Malhi GS (2007). The cognitive and neurophysiological basis of emotion dysregulation in bipolar disorder. J Affect Disorders 103: 29-42.

Haddjeri N, Szabo ST, de Montigny C, Blier P (2000). Increased tonic activation of rat forebrain 5-HT(1A) receptors by lithium addition to antidepressant treatments. Neuropsychopharmacology 22: 346-356.

Hajek T, Carrey N, Alda M (2005). Neuroanatomical abnormalities as risk factors for bipolar disorder. Bipolar Disord 7: 393-403.

Haznedar MM, Roversi F, Pallanti S, Baldini-Rossi N, Schnur DB, LiCalzi EM et al (2005). Fronto-thalamo-striatal gray and white matter volumes and anisotropy of their connections in bipolar spectrum illnesses. Biol Psychiatry 57: 733-742.

Holmes CJ, Hoge R, Collins L, Woods R, Toga AW, Evans AC (1998). Enhancement of MR images using registration for signal averaging. J Comput Assist Tomogr 22: 324-333.

Houenou J, Wessa M, Douaud G, Leboyer M, Chanraud S, Perrin M et al (2007). Increased white matter connectivity in euthymic bipolar patients: diffusion tensor tractography between the subgenual cingulate and the amygdalo-hippocampal complex. Mol Psychiatry 12: 1001-1010.

Jiang H, van Zijl PCM, Kim J, Pearlson GD, Mori S (2006). DtiStudio: resource program for diffusion tensor computation and fiber bundle tracking. Comput Methods Programs Biomed 81: $106-116$.

Jones DK, Symms MR, Cercignani M, Howard RJ (2005). The effect of filter size on VBM analyses of DT-MRI data. Neuroimage 26: $546-554$

Kabasawa H, Masutani Y, Abe O, Aoki S, Ohtomo K (2008). Quantitative diffusion tensor analysis using multiple tensor ellipsoids model and tensor field interpolation at fiber crossing. Acad Radiol 15: 84-92.

Kaplan W (2003). Advanced Calculus, 5th edn. Addison Wesley: Boston.

Kent JT, Bibby JM, Marida KV (1980). Multivariate Analysis: Probability and Mathematical Statistics. Academic Press: San Diego.

Kieseppa T, van Erp TG, Haukka J, Partonen D, Cannon TD, Poutanen VP et al (2003). Reduced left hemispheric white matter volume in twins with bipolar I disorder. Biol Psychiatry 54: 896-905.

Konarski JZ, McIntyre RS, Grupp LA, Kennedy SH (2005). Is the cerebellum relevant in the circuitry of neuropsychiatric disorders? J Psychiatry Neurosci 30: 178-186.

Makris N, Kennedy DN, McInerney S, Sorenson AG, Wang R, Caviness Jr VS et al (2005). Segmentation of subcomponents within the superior longitudinal fascicle in humans: a quantitative, in vivo, DT-MRI study. Cereb Cortex 15: 854-869.

Marchand WR, Lee JN, Thatcher GW, Jensen C, Stewart D, Dilda V et al (2007). A functional MRI study of a paced motor activation task to evaluate frontal-subcortical circuit function in bipolar depression. Psychiatr Res 155: 221-230.

Mills NP, DelBello MP, Adler CM, Strakowski SM (2005). MRI analysis of cerebellar vermal abnormalities in bipolar disorder. Am J Psychiatry 162: 1530-1532.

Moore PB, Shepherd DJ, Eccleston D, MacMillan IC, Goswami U, McAllister VL et al (2001). Cerebral white matter lesions in 
bipolar affective disorder: relationship to outcome. $\mathrm{Br} \mathrm{J}$ Psychiatry 178: 172-176.

Moorhead TW, McKirdy J, Sussmann JE, Hall J, Lawrie SM, Johnstone EC et al (2007). Progressive gray matter loss in patients with bipolar disorder. Biol Psychiatry 62: 894-900.

Monkul ES, Hatch JP, Sassi RB, Axelson D, Brambilla P, Nicoletti MA et al (2008). MRI study of the cerebellum in young bipolar patients. Prog Neuropsychopharm Biol Psychiatry 32: 613-619.

Norris SD, Krishnan KRR, Ahearn E (1997). Structural changes in the brain of patients with bipolar affective disorder by MRI: a review of the literature. Prog Neuropsychopharmacol Biol Psychiatry 21: 1323-1337.

Oouchi H, Yamada K, Sakai K, Kizu O, Kubota T, Ito $\mathrm{H}$ et al (2007). Diffusion anisotropy measurement of brain white matter is affected by voxel size: underestimation occurs in areas of crossing fibers. AJNR 28: 1102-1106.

Rajkowska G, Halaris A, Selemon LD (2001). Reductions in neuronal and glial density characterize the dorsolateral prefrontal cortex in bipolar disorder. Biol Psychiatry 49: 741-752.

Schmahmann JD (2000). The role of the cerebellum in affect and psychosis. J Neuroling 13: 189-214.

Skare S, Andersson JL (2001). On the effects of gating in diffusion imaging of the brain using single shot EPI. Magn Reson Imaging 19: $1125-1128$.

Smith SM (2002). Fast robust automated brain extraction. Hum Brain Mapp 17: 143-155.

Smith SM, Jenkinson M, Johansen-Berg H, Rueckert D, Nichols TE, Mackay C et al (2006). Tract-based spatial statistics: voxelwise analysis of multi-subject diffusion data. Neuroimage 31: 1487-1505.

Smith SM, Johansen-Berg H, Jenkinson M, Rueckert D, Nichols TE, Miller KL et al (2007). Acquisition and voxelwise analysis of multi-subject diffusion data with tract-based spatial statistics. Nat Protoc 2: 499-503.

Soares JC, Mann JJ (1997). The functional neuroanatomy of affective disorders. J Psychiatr Res 31: 393-432.

Song SK, Sun SW, Ramsbottom MJ, Chang C, Russell J, Cross AH (2002). Dysmyelination revealed through MRI as increased radial (but unchanged axial) diffusion of water. Neuroimage 17: 1429-1436.

Song SK, Yoshino J, Le TQ, Lin SJ, Sun SW, Cross AH et al (2005). Demyelination increases radial diffusivity in corpus callosum of mouse brain. Neuroimage 26: 132-140.

Stoll AL, Renshaw PF, Yurgelun-Todd DA, Cohen BM (2000). Neuroimaging in bipolar disorder: what have we learned? Biol Psychiatry 48: 505-517.

Strakowski SM, Adler CM, Holland SK, Mills NP, DelBello MP, Eliassen JC (2005a). Abnormal fMRI brain activation in euthymic bipolar patients during a counting Stroop interference task. Am J Psychiatry 162: 1697-1705.

Strakowski SM, DelBello MP, Adler C, Cecil DM, Sax KW (2000). Neuroimaging in bipolar disorder. Bipolar Disord 2: 148-164.

Strakowski SM, DelBello MP, Adler CM (2005b). The functional neuroanatomy of bipolar disorder: a review of neuroimaging findings. Mol Psychiatry 10: 105-116.

Strakowski SM, Wilson DR, Tohen M, Woods BT, Douglass AW, Stoll AL (1993). Structural brain abnormalities in first-episode mania. Biol Psychiatry 33: 602-609.

Sun Y, Zhang L, Johnston NL, Torrey EF, Yolken RH (2001). Serial analysis of gene expression in the frontal cortex of patients with bipolar disorder. Br J Psychiatry Suppl 41: s137-s141.

Szeszko PR, Ardekani BA, Ashtari M, Kumra M, Robinson DG, Sevy S et al (2005a). White matter abnormalities in first-episode schizophrenia or schizoaffective disorder: a diffusion tensor imaging study. Am J Psychiatry 162: 602-605.

Szeszko PR, Ardekani BA, Ashtari M, Malhotra AK, Robinson DG, Bilder RM et al (2005b). White matter abnormalities in obsessive-compulsive disorder: a diffusion tensor imaging study. Arch Gen Psychiatry 62: 782-790.

Szeszko PR, Robinson DG, Ashtari M, Vogel J, Betensky J, Sevy S et al (2008). Clinical and neuropsychological correlates of white matter abnormalities in recent onset schizophrenia. Neuropsychopharmacology 33: 976-984.

Taylor WD, Payne ME, Krishnan KRR, Wagner HR, Provenzale JM, Steffens DC et al (2001). Evidence of white matter tract disruption in MRI hyperintensities. Biol Psychiatry 50: $179-183$.

Tkachev D, Mimmack L, Ryan MM, Wayland M, Freeman T, Jones PB et al (2003). Oligodendrocyte dysfunction in schizophrenia and bipolar disorder. Lancet 362: 798-805.

Uranova NA, Vostrikov VM, Orolovskaya DD, Rachmanova VI (2004). Oligodendroglial density in the prefrontal cortex in schizophrenia and mood disorders: a study from the Stanley Neuropathology Consortium. Schizophr Res 67: 269-275.

Wessa M, Houenou J, Paillère-Martinot ML, Berthoz S, Artiges E, Leboyer $\mathrm{M}$ et al (2007). Fronto-striatal overactivation in euthymic bipolar patients during an emotional go/nogo task. Am J Psychiatry 164: 638-646.

Yurgelun-Todd DA, Silveri MM, Gruber SA, Rohan ML, Pimentel PJ (2007). White matter abnormalities observed in bipolar disorder: a diffusion tensor imaging study. Bipolar Disord 9: 504-512.

Zhang Y, Brady M, Smith S (2001). Segmentation of brain MR images through a hidden Markov random field model and the expectation maximization algorithm. IEEE Trans Med Imaging 20: $45-57$. 